\title{
An efficient and simple co-culture method for isolating primary human hepatic cells: Potential application for tumor microenvironment research
}

\author{
WEI DONG ${ }^{1}$, AIGUO LU $^{3}$, JINGKUN ZHAO ${ }^{2}$, SHUAI YIN ${ }^{2}$, BAOCHI OU ${ }^{1,3}$ and HAO FENG ${ }^{1,3}$ \\ ${ }^{1}$ Heart Center, Shanghai Children's Medical Center, Shanghai Jiao Tong University School of Medicine, \\ Shanghai 200127, P.R. China; ${ }^{2}$ Department of General, Visceral, Transplantation and Vascular Surgery, \\ University Hospital of Munich (Ludwig Maximilian University of Munich), D-81377 Munich, Germany; ${ }^{3}$ Department of \\ General Surgery, Ruijin Hospital, Shanghai Jiao Tong University School of Medicine, Shanghai 200025, P.R. China
}

Received February 22, 2016; Accepted April 7, 2016

DOI: $10.3892 /$ or.2016.4979

\begin{abstract}
Co-cultivation of non-parenchymal cells (NPCs) and tumor cells from the same donor is important for metastatic cancer research. This study aimed to optimize a protocol for liver NPC isolation. Two novel 3D organotypic co-culture models for hepatocyte, endothelial cell (EC) and Kupffer cell (KC) isolation were used. Long-term cell co-culture, density gradient centrifugation and magnetic-activated cell sorting (MACS) were established. ECs were isolated from the co-culture system; the purity of the ECs was $92 \pm 1.2 \%$. The island-like shape of hepatocytes was noted in the 3D co-culture system, and spindle cells were found in the rest space. Immunofluorescence analysis showed a net structure; the connective tissue was positively stained with VE-cadherin or CD68, which were ECs and KCs/macrophages. KCs were enriched in this system and separated by using selective adherence to plastic. $\mathrm{Clec}_{4} \mathrm{f}^{+} \mathrm{KC}$ consisted of $87 \pm 6.3 \%$ of these cells. Heterogeneous endothelium populations were detected, including sinusoid ECs, microvascular ECs and hepatic lymphatic vessel epithelial cells. In addition, hepatic progenitor cells were isolated and differentiated into hepatoblasts. Dendritic cells (DCs), invariant natural killer T (iNKT) cells were further separated by density gradient centrifugation and magnetic bead sorting. In the present study, high protein expression levels of desmin and GFAP were observed in the hepatic stellate cells (HSCs). Most of the HSCs were $\alpha$-SMA-positive cells, which underlined the identity of activated HSCs. Intrahepatic human biliary epithelial cells (hBECs) were semi-purified by centrifugation on a Percoll gradient and were
\end{abstract}

Correspondence to: Dr Hao Feng, Department of General Surgery, Ruijin Hospital, Shanghai Jiao Tong University School of Medicine, Shanghai 200025, P.R. China

E-mail: hao.feng@med.uni-muenchen.de

Key words: liver, isolation, non-parenchymal cells, co-culture, 3D cell culture, protocol further immunopurified. In conclusion, we provide an efficient long-term culture method to obtain liver NPCs in sufficient number and purity.

\section{Introduction}

An extensive body of clinical data and experimental research has confirmed Stephen Paget's original 'seed and soil' hypothesis that proposed that the organ-preference patterns of tumor metastasis are the product of favorable interactions between metastatic tumor cells (the 'seed') and their organ microenvironment (the 'soil') $(1,2)$. The liver is the second most common site of metastasis (spread of cancer), after the lymph nodes. Liver metastases have been found in $30-70 \%$ of patients who are dying of cancer (3). Breast cancer, gastrointestinal cancer, melanoma and pancreatic cancer all might spread to the liver, especially colorectal cancer. Unfortunately, only $20 \%$ of patients with colorectal liver metastases present liver-confined resectable disease and/or are candidates for major surgical operation (depending on comorbidities) (4,5). Importantly, 5 -year survival rates are reported to be in the range of $40-58 \%$ (6). Based on the above analysis, it is important to investigate the liver environment and establish co-culture models for cancer research. Discovery of an efficient isolating method is definitely the first step, not only for hepatocytes but also for non-parenchymal cells (NPCs) such as liver sinusoid endothelial cells (LSECs), hepatic stellate cells (HSCs) and liver resident immune cells, including Kupffer cells (KCs), invariant natural killer T (iNKT) cells as well as dendritic cells (DCs). However, successful and consistent isolation of primary human hepatic cells remains a challenge for both cell-based therapeutics and laboratory research (7). Herein, a relatively synchronous (7-14 days for each cell type) and efficient method for isolating primary human hepatocytes (PHHs) and liver NPCs from surgical specimens is described.

\section{Materials and methods}

Tissue specimens. Liver wedges were obtained from non-diseased tissue removed at surgical resection for liver tumor, liver 
metastasis from colorectal carcinoma or fresh transplanted specimens ( $\mathrm{n}=21$; weight, 20-83 g). The surgical procedures were carried out at the University Hospital of Munich and Shanghai Jiao Tong University School of Medicine. All patients provided written documentation of informed consent. The human liver pieces used in this study were acquired with consent for governance through the Human Tissue and Cell Research Foundation (http://www.htcr. de/english/home.html), a state-controlled non-profit foundation (ref. no. Antrags-Nr. 2014-01). After a pathologist removed what was required for diagnosis, liver pieces were collected from the remaining tissue. The tissue sectioned off by the pathologist was morphologically a healthy tissue obtained from resection margins after liver resection. The study conforms to the ethical guidelines of the Declaration of Helsinki and was approved by the Human Tissue and Cell Research Foundation and the Ethics Committee of the University Hospital of Munich and Shanghai Jiao Tong University School of Medicine.

Isolation of PHHs and NPCs. Liver cells were prepared according to a modified 'two-stage' perfusion technique described by Seglen (8) and isolated as previously described (9). Following 'two-stage' collagenase (batch no. NB 4G, cat. no. 17465; SERVA Electrophoresis GmbH, Germany) procedure and manual dissociation of the liver wedge, the suspension was passed through a sterile nylon mesh of $210 \mu \mathrm{m}$ (neoLab 4-1413) followed by a sterile nylon mesh of $70 \mu \mathrm{m}$ (neoLab 4-1419) (both from neoLab Migge GmbH, Germany). Suspensions were then washed once at $50 \mathrm{x} \mathrm{g}$ for $5 \mathrm{~min}$ at $4^{\circ} \mathrm{C}$ in cold storage solution (CSS; Hepacult GmbH, Germany). The pellet was washed two more times for hepatocyte enrichment. The NPC-containing cell suspension, as the supernatant obtained after the first wash, was further used to isolate KCs, LSECs, HSCs, DCs and iNKT cells. The procedure is schematically illustrated in Fig. 1. In detail, the supernatant was collected in $200 \mathrm{ml}$ polypropylene centrifuge tubes (cat. no. 352075; BD Biosciences) and pelleted by $600 \mathrm{rpm}$ centrifugation (Eppendorf 5810R; Eppendorf) for $5 \mathrm{~min}$ at $4^{\circ} \mathrm{C}$.

1) The pellet was then resuspended and seeded in 12-well ultra-low attachment plates (Sigma-Aldrich, Germany) at a density of $1.5-2 \times 10^{7}$ viable cells $/ \mathrm{cm}^{2}$ by using Dulbecco's modified Eagle's medium (DMEM)/Ham's F-12 (Gibco Life Technologies, Germany). This pellet contained the remaining hepatocytes and $\mathrm{KCs}$; the mixture was co-cultured for 2-3 weeks with $10 \mu \mathrm{g} / \mathrm{ml} \mathrm{HGF}$ (red arrow, Fig. 1). The cells were collected at 14-21 days from each well and washed three times, and then the cells were transferred into $100-\mathrm{mm}$ non-tissue culture grade plastic dishes. Incubation was carried out for $30 \mathrm{~min}$ at $37^{\circ} \mathrm{C}$ in an atmosphere of $5 \% \mathrm{CO}_{2}$ and $\sim 95 \%$ air. Macrophages readily attached to the non-tissue culture grade plastic dishes under this incubation process, whereas other types of cells did not. Two milliliters of trypsin solution was added into the dish and incubated for $10 \mathrm{~min}$ at $37^{\circ} \mathrm{C}$ in an atmosphere of $5 \% \mathrm{CO}_{2}$ and $\sim 95 \%$ air. Then $10 \mathrm{ml}$ of the growth medium were added and the cells were gently scraped. The collected $\mathrm{KCs}$ were then centrifuged at 1,500 rpm for $5 \mathrm{~min}$, and propagated in $75-\mathrm{cm}^{2}$ flasks (red dashed line in Fig. 1).

2) The supernatant washed with CSS was collected in a 50-ml polystyrene conical Falcon tube (BD Biosciences) and pelleted at $1,500 \mathrm{rpm}$ for $7 \mathrm{~min}$ at $4^{\circ} \mathrm{C}$. After three times washing, the pellet was seeded in collagen I-coated empty tissue culture 75- $\mathrm{cm}^{2}$ flasks or 6-well Corning BioCoat Collagen I Multiwell Plates (both from Corning, Inc.) with DMEM/F-12 and EGF, $10 \% \mathrm{FBS}, 100 \mathrm{U} / \mathrm{ml}$ penicillin, $0.1 \mathrm{mg} / \mathrm{ml}$ streptomycin, and $2 \mathrm{mM}$ L-glutamine for hepatic microvascular endothelial cell (EC) isolation (purple arrow, Fig. 1). The assessment of yield and viability was made by using a hemocytometer-based trypan blue exclusion assay. DMEM/F-12 plus $0.1 \mathrm{ng} / \mathrm{ml}$ recombined human EGF and $90 \mu \mathrm{g} / \mathrm{ml}$ heparin $(10 \mathrm{ng} / \mathrm{ml}$ insulin-like growth factor was also optimal) were used from day 4 which lasted for $\sim 7-8$ days. On day 7, the hepatocytes were detached by trypsin (PAA) for 4-5 min and the procedure was observed by microscopy. Hepatocytes were then washed out, and the rest were microvascular epithelial cells (Fig. 2).

3) Or the pellet was processed as mentioned in step 1. The supernatant was placed in 50-ml Falcon tubes after washing once with RPMI-1640 medium by centrifugation at $800 \mathrm{x}$ g for $5 \mathrm{~min}$. The cells were then resuspended in $8 \mathrm{ml} \mathrm{30 \%} \mathrm{Percoll}{ }^{\mathrm{TM}}$ PLUS/Percoll (GE Healthcare Life Sciences, Freiburg, Germany), placed in 15-ml polystyrene conical centrifuge tube (BD Biosciences) and overlaid with $3 \mathrm{ml} 70 \%$ Percoll solution. After being centrifuged at 2,500 rpm for $20 \mathrm{~min}$ at room temperature with decreased acceleration and without breaks, the various cell types were arranged according to their density. HSCs were enriched in an upper cell layer, whereas macrophages/KCs, oval cells and LSEC fractions were floating between 50 and 60\% Percoll. DCs, monocytes and lymphocytes were enriched in the interphase of 60 and $70 \%$ Percoll. Mature intrahepatic biliary epithelial cells were floating on the $70 \%$ Percoll. Cell fractions were collected separately by pipetting (10)

4) For the immune cell enrichment, the monocyte fraction was pelleted, resuspended in $1 \mathrm{ml}$ of $10 \mathrm{X} \mathrm{RBC}$ lysis buffer and transfered to a new tube. The cell suspension was allowed to stay for 3 min at room temperature to lyse RBCs, and was then diluted with $9 \mathrm{ml}$ RPMI-1640 medium, centrifuged at $800 \mathrm{x} \mathrm{g}$ for $5 \mathrm{~min}$ at $4^{\circ} \mathrm{C}$ and the cells were washed twice with $10 \mathrm{ml}$ of RPMI-1640 medium. The live cell number was counted and adjusted to a cell concentration of $1 \times 10^{6}$ cells $/ \mathrm{ml}$ and placed in $75-\mathrm{cm}^{2}$ flasks after being washed three times. A total of $100 \mathrm{ng} / \mathrm{ml} \alpha$-galactosylceramide ( $\alpha$-Galcer; Abcam, UK) was added $8 \mathrm{~h}$ after seeding. The medium containing $\alpha$-Galcer was changed every 2-3 days. DCs were induced and enriched in the first $48 \mathrm{~h}$, being replaced by monocyte-induced macrophages after 72 h. On day 7-10, the supernatant was collected and washed three times. After being adjusted to a cell concentration of $1 \times 10^{6}$ cells $/ \mathrm{ml}$ by adding FACS staining buffer, the cells were labeled with iNKT (clone 6B-11) microbeads (human TCR V $\alpha 24-J \alpha 18$ combined with V $\beta 11$; Miltenyi Biotec GmbH, Germany) according to the manufacturer's instructions. Cells were applied onto LS magnetic-activated cell sorting (MACS) columns (Miltenyi Biotec $\mathrm{GmbH}$ ) twice, which were placed within the magnetic field of a MACS separator and washed three times with MACS buffer (Miltenyi Biotec $\mathrm{GmbH}$ ). V $\alpha 24-J \alpha 18$ combined with V $\beta 11$-positive iNKT cells were eluted from the columns, and the viable iNKT cells were counted and propagated in flasks.

5) DCs were enriched on day 2 or 3 as attached cells. However, other monocytes were in the suspension. These cell suspensions were collected and were propagated in another 


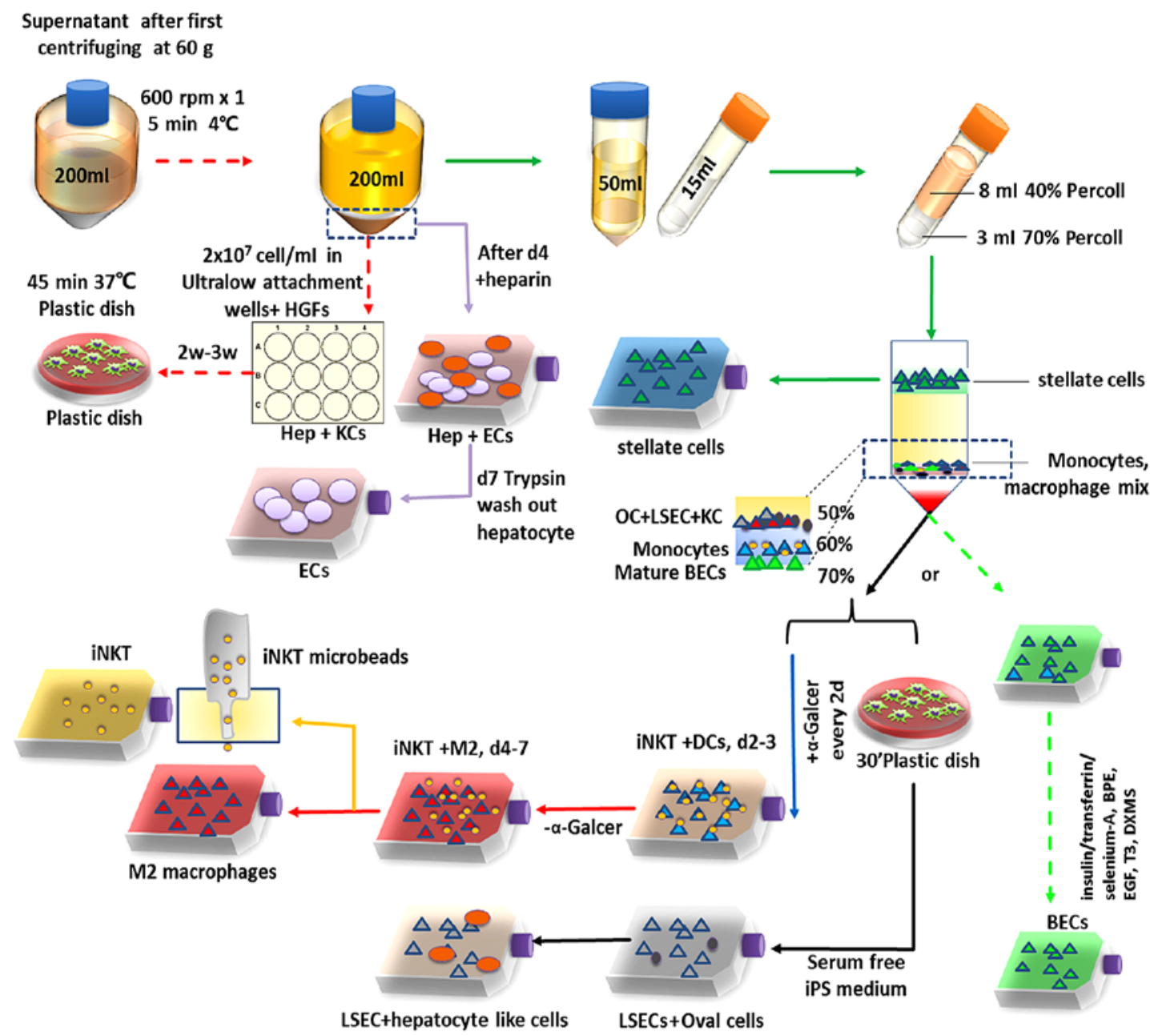

Figure 1. Schematic diagram showing the steps used to isolate the NPCs from the supernatant which was collected from the first centrifuge after the perfusion method. NPCs, non-parenchymal cells; KCs, kupffer cells; DCs, dendritic cells; ECs, endothelial cells; Mac, macrophages; iNKT cells, invariant natural killer T cells; LSECs, liver sinusoid endothelial cells; BECs, biliary epithelial cells

flask. The remaining cells were detached by trypsin (PAA) and were incubated with human IgG $\left(1 \mu \mathrm{g} / 10^{6} \mathrm{ml}\right.$; BioLegend, Inc, San Diego, CA, USA) to block non-specific binding to Fc $\gamma$ receptors for $15 \mathrm{~min}$. The cells were then labeled with CD1c microbeads. CD1 $\mathrm{c}^{+} \mathrm{DCs}$ were propagated in culture plates (blue arrow, Fig. 1) after magnetic sorting.

6) Cell suspension as mentioned in step 4 was collected and propagated in another flask as step 4 . The former flasks which contained the attached cells were refilled with $20 \mathrm{ml}$ completed growth medium. M2-differentiated macrophages were enriched on day 4-7, iNKT cells was also separated here (orange arrow, red arrow, Fig. 1). Alternatively, culture plates coated by the attached cells from the last step were cultivated with DMEM containing IL-4 and GM-CSF for 5 days, and M2-differentiated macrophages were harvested.

The macrophage/KC fraction was pelleted and $\mathrm{KCs}$ were also labeled with APC-conjugated anti-Clec4f antibody (LS-C233943, LifeSpan Biosciences, Inc., USA) for $45 \mathrm{~min}$ at $10 \mu \mathrm{l} / 100 \mu \mathrm{l}$ after a 15 -min incubation of human IgG blocking, followed by $10 \mu \mathrm{l} / 100 \mu \mathrm{l}$ anti-APC-microbead incubation (Miltenyi Biotec $\mathrm{GmbH}$ ) and magnetic cell sorting according to the manufacturer's instructions. Cells were resuspended in growth medium and seeded at a sufficient density of $1-2 \times 10^{6} \mathrm{cells} / \mathrm{cm}^{2}$.
7) For the stem cell enrichment, the fraction between 50 and $60 \%$ Percoll was pelleted and seeded in a $75-\mathrm{cm}^{2}$ collagen I-coated flask after being washed three times and cultivated in a plastic dish for $30 \mathrm{~min}$ to eliminate KCs. Serum-free iPSC medium plus EGF was placed for the cell culture. ECs were proliferated from day 3. Hepatic progenitor cell-like cells differentiated to hepatocytes surrounding the EC niches from day 5 .

8) For the intrahepatic biliary epithelial cell isolation and purification, the fraction floating on $70 \%$ Percoll was seeded in $75-\mathrm{cm}^{2}$ collagen I-coated empty tissue culture flasks (Corning, Inc.). Cells were grown in medium containing DMEM/ F-12 (Sigma-Aldrich, St. Louis, MO, USA) supplemented with fetal bovine serum (Cellgro; Mediatech, Inc. Manassas, VA, USA), penicillin/streptomycin, vitamin solution, MEM solution, CD lipid concentrate, L-glutamine, insulin/transferrin/selenium-A, bovine pituitary extract, epidermal growth factor, 3,3',5-triiodo-L-thyronine and dexamethasone.

Identification of cell populations. Morphological characteristics were determined by phase contrast images acquired with an Olympus IX50 microscope (Olympus, Germany). Cells cultured in 8-well chamber slides (Falcon; BD Biosciences, Germany) and culture inserts (Ibidi GmbH, Martinsried, 
Germany) were washed twice with cold Tris-buffered saline (TBS), $\mathrm{pH} 7.4$, fixed with $4 \%$ paraformaldehyde for $10 \mathrm{~min}$, permeabilized with $0.1 \%$ Triton $\mathrm{X}-100$ for $5 \mathrm{~min}$ (for the staining of extracellular markers, permeabilization was not necessary), blocked with 5\% BSA, incubated with the indicated primary antibodies: rabbit anti-human Clec4f (Sigma-Aldrich, Germany), mouse anti-human CD68 (Sigma-Aldrich), rabbit anti-human epithelial cell adhesion molecule (EpCAM, Cell Signaling Technology, Inc., UK), anti-GFAP and anti-desmin (Sigma-Aldrich), anti- $\alpha$-SMA (Abcam), anti-OV-6 (Santa Cruz Biotechnology, Inc., Paso Robles, CA, USA), cytokeratin-19 (CK-19; Santa Cruz Biotechnology, Inc.), rabbit anti-human VE-cadherin (Cell Signaling Technology, Inc.) and APC-iNKT (Miltenyi Biotec $\mathrm{GmbH}$ ) at $4^{\circ} \mathrm{C}$ overnight followed by anti-rabbit Alexa Fluor 488 and anti-mouse Alexa Fluor 568-conjugated secondary antibodies (Life Technologies, Germany). The cells were then stained with anti-fade DAPI (Life Technologies) for nuclear staining, and the images were acquired with an Olympus Axion microscope (Olympus, Japan).

$3 D$ cell culture. Two 3D cell culture models were used in this study, ultra-low attachment plates (Sigma-Aldrich) and HDP1096 Perfecta3D ${ }^{\circledR}$ 96-Well Hanging Drop Plates (3D Biomatrix, Inc.). For the hanging drop plates, the pellet collected from step 1 was washed three times and a 100 cell $/ \mu 1$ cell suspension was prepared. Hanging drops were formed by pipetting $45 \mu \mathrm{l}$ of cell suspension into each well from the top side of the plate. Hanging drops were formed on and confined to the bottom of the plate. The lid was placed on the plate and the assembly was placed into a tissue culture incubator. Within hours, individual cells started to aggregate and eventually formed into spheroids. Media exchange followed the protocol supplied by 3D Biomatrix, Inc. DMEM/F-12 with HGF, 10\% FBS, $100 \mathrm{U} / \mathrm{ml}$ penicillin, $0.1 \mathrm{mg} / \mathrm{ml}$ streptomycin, and $2 \mathrm{mM}$ L-glutamine was used for culture maintenance.

Statistical analysis. All continuous values are expressed as means \pm SD and all experiments were repeated three times. Immunofluorescence staining of cell type-specific markers was performed in five independent experiments. For quantitative analysis, DAPI-stained, marker-positive and -negative cells were counted in 10 independent images per cell type at $\mathrm{x} 20$ magnification. The results were subjected to a non-parametric Mann-Whitney U test. A paired Student's t-test was also used to analyze the intragroup differences. All statistical analyses were conducted using StatView 5.0 for Windows (SAS Institute, Inc., Cary, NC, USA). Student's t-test was also used to test differences in cell viability assays. $\mathrm{P}<0.05$ was considered statistically significant.

\section{Results}

Hepatic (microvascular) EC isolation, primary culture and subculture based on the co-culture system. The supernatant from the first washing was centrifuged, the pellet was resuspended and plated in collagen I-coated flasks and processed as detailed in 'Materials and methods'. Fig. 2A and B show representative images of $\mathrm{PHH}$ and microvascular ECs, in culture on days 4 and 5 after isolation. The hepatocytes formed an island-like shape with an irregular outline. In the rest space proliferated ECs were noted until day 7-9 (Fig. 2B). Fig. 2C and D shows the morphological features of the hepatocyte and EC co-culture system 6 and 7 days after isolation. The ECs exhibited cobblestone morphologic characteristics, which resembled the shape of microvascular epithelial cells (11). The cultured ECs were harvested as early as day 8, by detachment of hepatocytes. After a 4-5-min trypsin treatment, the hepatocytes were soon detached, however ECs were still attached (Fig. 2E). The number of ECs reached maximal levels on day 12-14, when the cells formed a flat cell sheet and tight conjunction on the plate surface (Fig. 2F). When the colonies of ECs reached $80 \%$ confluency, the cells were detached by trypsin for 8-10 min and subcultured in new collagen I-coated tissue culture flasks. The purity of the ECs was $92 \pm 1.2 \%$ as characterized by VE-cadherin and EpCAM.

$K C$ isolation and purification based on the $3 D$ co-culture system. Similar to what was observed in the 2D co-culture system for EC isolation, the island-like shape of hepatocytes was also observed in the 12- or 96-well 3D co-culture system (Fig. 3A). Spindle cells were found in the rest space (Fig. 3B). Primary KCs, prepared from the 3D co-culture system and plastic dish attachment as described in step 1, formed typical irregular morphology of both a stretched and an oval shape (12). Immunofluorescence staining was performed using the same methods as below. The medium in each well was carefully removed and gently refilled with embedding medium for cryosectioning (Leica, Germany), followed by freezing of the plates. The $3 \mathrm{D}$ co-culture model then underwent the immunofluorescence analysis. A net structure was found, and the connecting tissue was positively stained with VE-cadherin, CD68 or Clec4f (Fig. 3C-E), which were ECs and $\mathrm{KCs} /$ macrophages. $\mathrm{Clec} 4 \mathrm{f}^{+} \mathrm{KCs}$ were less than the $\mathrm{CD} 68^{+}$ macrophages in the $3 \mathrm{D}$ organotypic co-culture models after whole-mount staining by two-photon microscope (Carl Zeiss, Germany). In addition, the cells collected from the other wells were collected, centrifuged and washed three times. After that, KCs were enriched using the isolation method above (step 1). A total of $87 \pm 6.3 \%$ of these cells was Clec4f-positive (Fig. $6 \mathrm{G}$ and $\mathrm{M}$ ), which were $\mathrm{KCs}$ rather than peripheral derived macrophages (13).

LSEC isolation and heterogeneous endothelium populations. The morphology of LSECs was different from the microvascular ECs $(14,15)$ (Fig. 4A and C), although both were VE-cadherin ${ }^{+}$ $\mathrm{EpCAM}^{+}$(Fig. 4B and D), especially when observed in the co-culture system of ECs and LSECs (Fig. 4E). In addition, the LSECs tended to form hepatic EC niche when co-cultivated with hepatocytes or NPCs (Fig. 4F). However, it was difficult to tell the differences between the hepatic microvascular ECs and ECs from the hepatic lymphatic vessels (Fig. 6K) according to the morphology. Lymphatic vessel endothelial hyaluronan receptor-1 (LYVE-1) unequivocally distinguishes lymphatic from blood microvascular endothelium (16), as assessed by immunostaining with the anti-LYVE-1 antibody.

Hanging drop models for 3D hepatocyte culture. Spheroid formation time was 48-72 $\mathrm{h}$ and lasted for 9-12 days. Sponges were observed from day 10-12, which were formed by ECs 

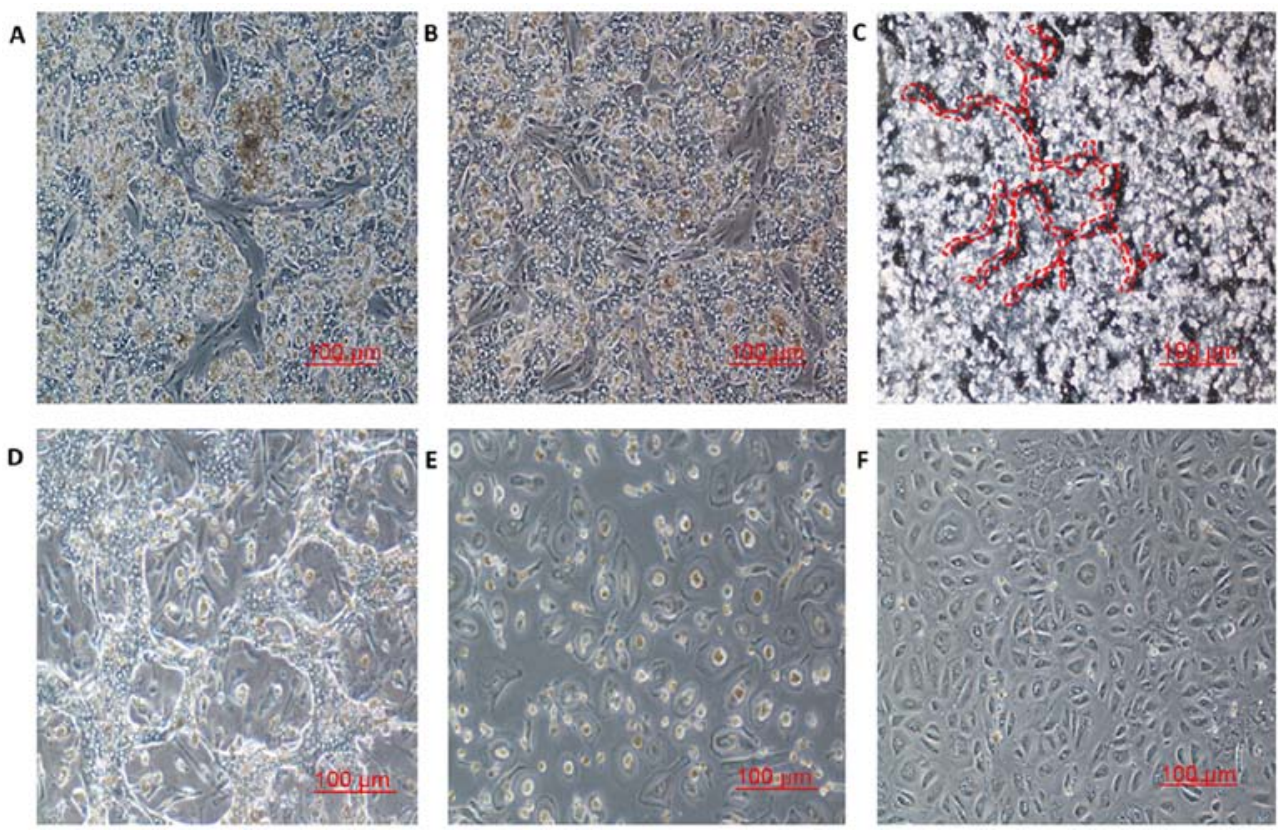

Figure 2. The co-culture system of ECs and hepatocytes from the pellet of the second wash of the supernatant in a collagen-coated flask when heparin was added to the medium from day 3 to 4. ECs were proliferated from (A) day 3 and (B) day 4. (C) From day 5, the structure of hepatocyte 'island' separated by LSECs was detected (the red dashed lines show the outline of sinusoid-like structure). (D) ECs enriched around day 7. (E and F) After short term (4-min) digestion by trypsin, the ECs were separated and enriched in serum-free medium with heparin after $72 \mathrm{~h}$. ECs, endothelial cells; LSECs, liver sinusoid endothelial cells.
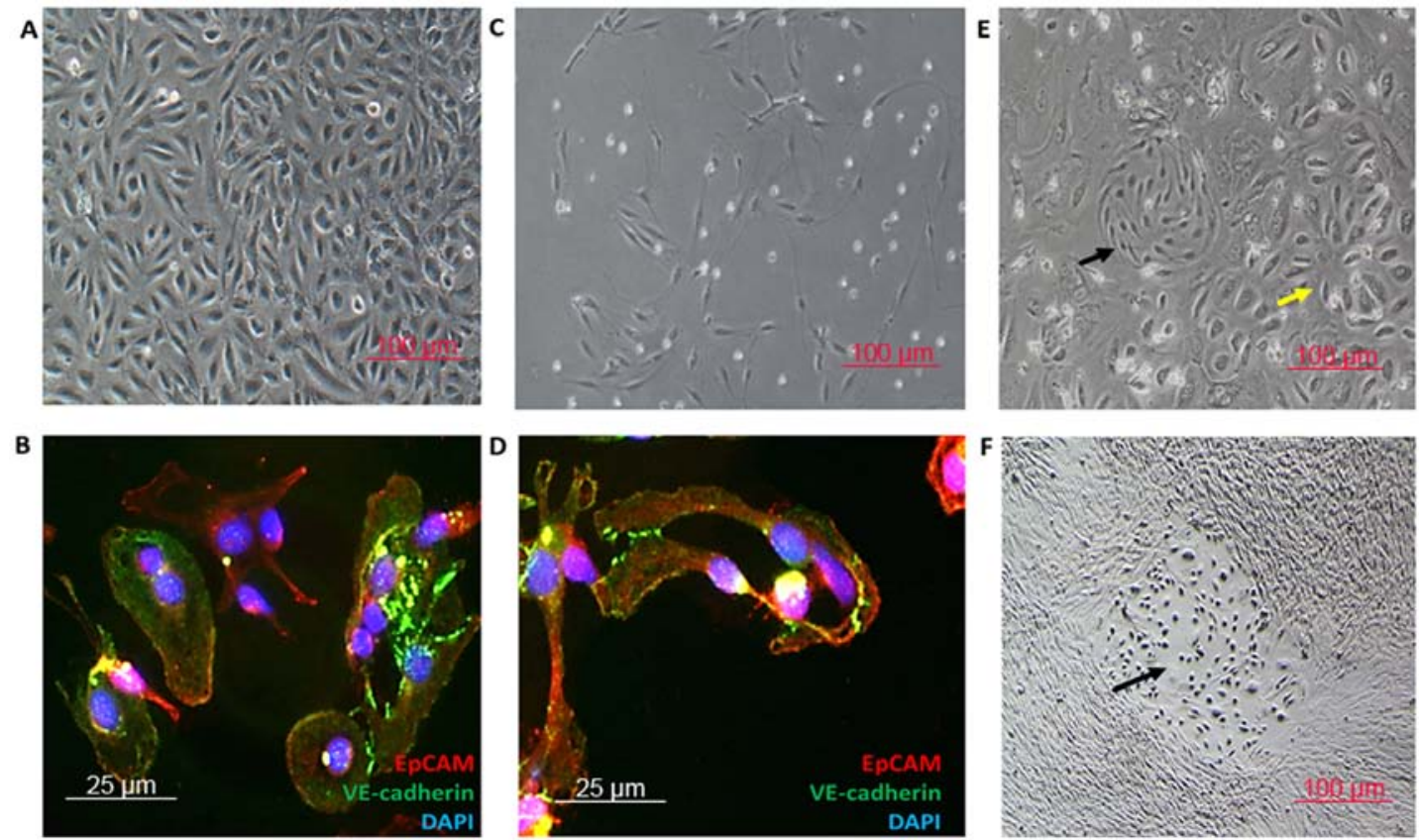

Figure 3. Heterogeneous endothelium population. (A and B) The morphology and immunophenotypic staining of microvascular ECs and (C and D) LSECs (red, EpCAM; green, VE-cadherin). (E) LSECs (black arrow) formed hepatic EC niches in the co-culture model (yellow arrow, microvascular ECs). (F) Morphological characteristics were determined by phase contrast microscopy and DIC microscopy. ECs, endothelial cells; LSECs, liver sinusoid endothelial cells.

and hepatocytes. VE-cadherin ${ }^{+}$ECs not only were detected in the sponges but also in the spheroid. According to the color variations and immunofluorescence, the spheroid was formed by three layers. Blood cells were the major source of the core, hepatocytes formed the second layer and maintained the capability of regeneration. However, ECs and other NPCs constituted the cover of the spheroid (Fig. 5).
Characterization of other NPCs. The complete yield of the HSCs, separated from the NPC suspension by density gradient centrifugation, was seeded into a $75-\mathrm{cm}^{2}$ tissue culture flask using stellate cell growth medium (cat. no. 5301; ScienCell Research Laboratories, Inc.). Once $90 \%$ confluency was reached, the cells were detached by trypsin and seeded into plastic dishes using DMEM supplemented with 10\% FBS, 

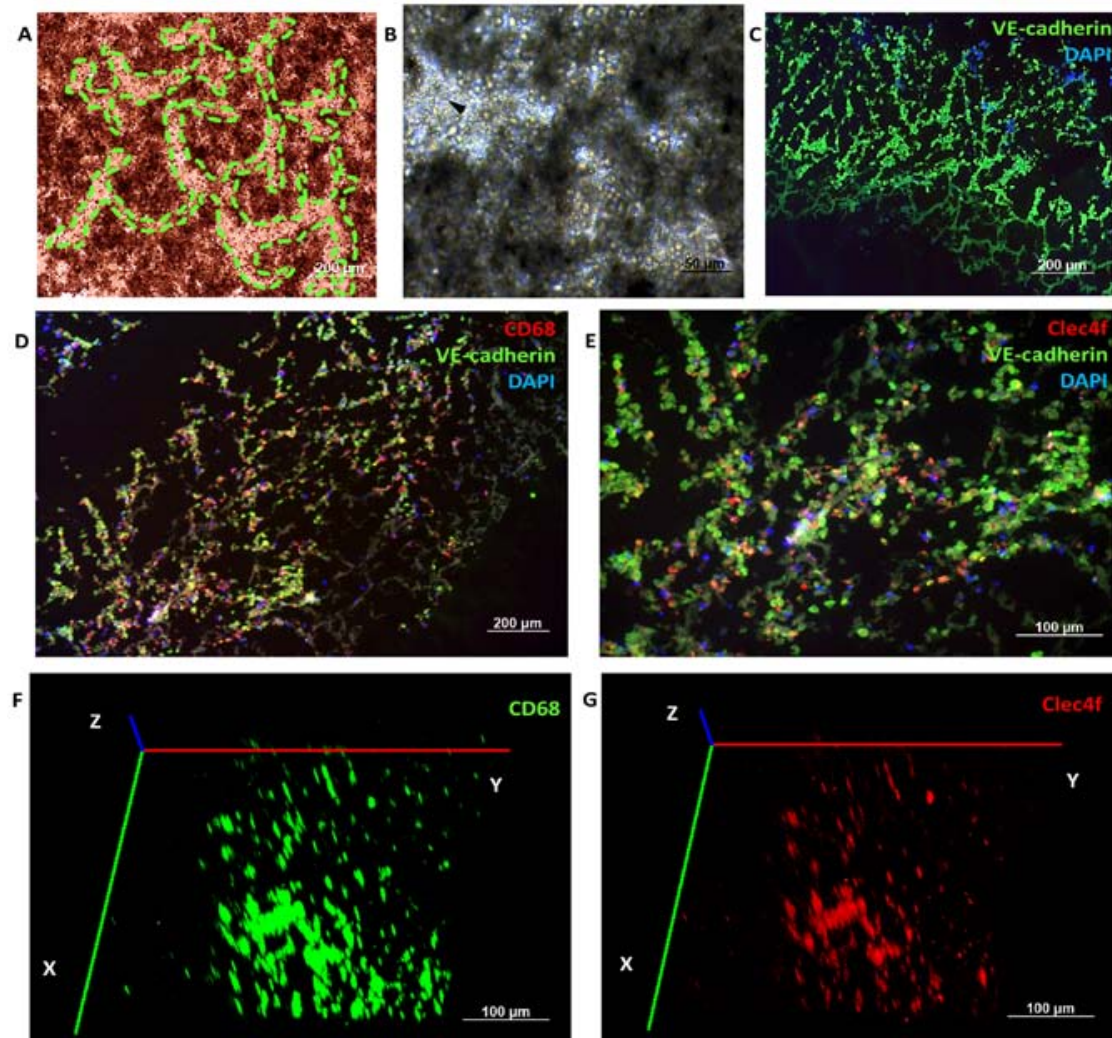

Figure 4. Ultra-low attachment plates were used for the 3D co-culture system. (A) Liver sinusoid-like structure were observed in the 3D culture system (the green dashed lines show the outline of liver sinusoid-like structure). (B) NPCs especially KCs (black arrow) were enriched in the liver sinusoid-like structure near the hepatocyte 'islands'. (C) The net structure was observed under immunofluorescence microscopy when we made the cryosection of the system (green, albumin; blue, DAPI). (D and E) KCs were observed in the net structure (green, VE-cadherin; red, CD68 or Clec4f; blue, DAPI). (G) Clec4f ${ }^{+}$KCs were less than the (F) $\mathrm{CD}^{+} 8^{+}$macrophages in the 3D organotypic co-culture models after whole-mount staining by two-photon microscopy (Carl Zeiss, Germany). NPCs, non-parenchymal cells; KCs, Kupffer cells.

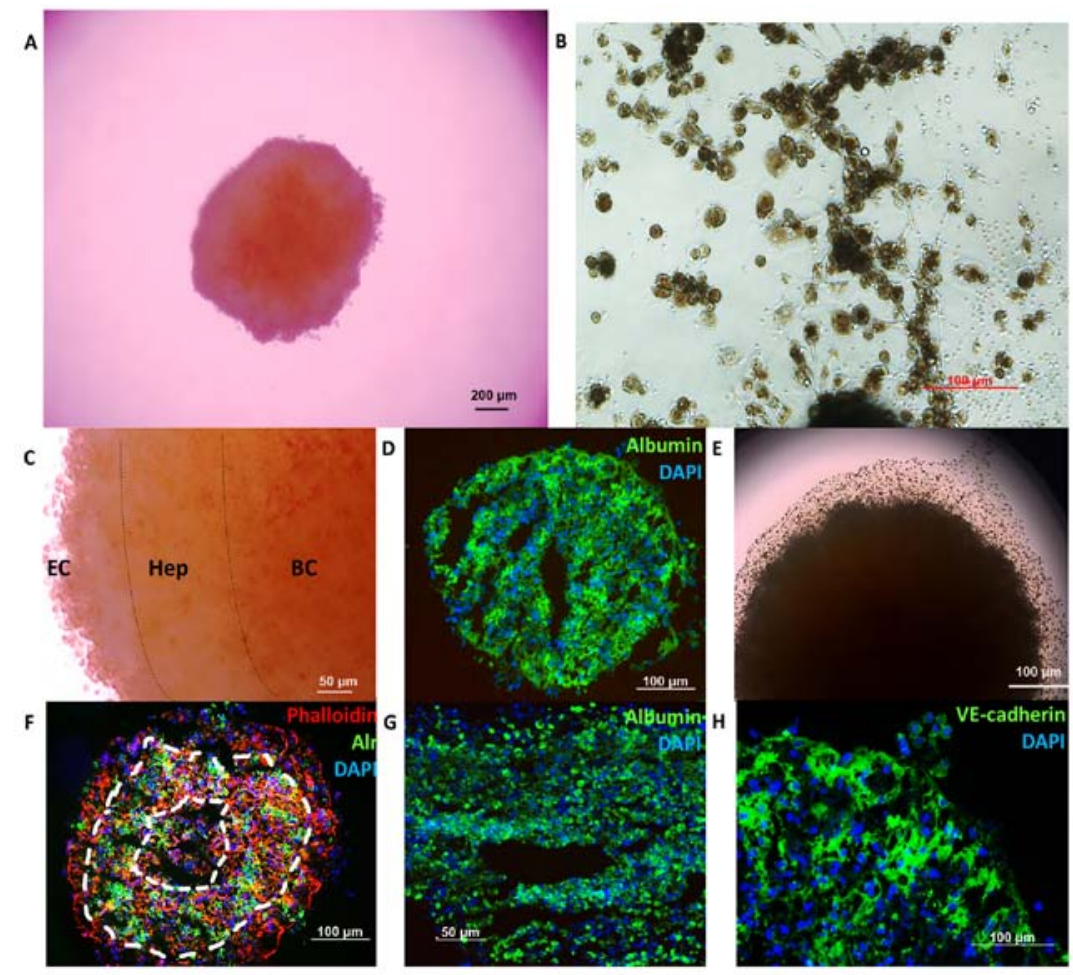

Figure 5. (A) 3D hepatocyte spheroid was performed in hanging drop plates. (C) The spheroid was formed by three layers. Blood cells (BC) were the major source of the core, hepatocytes (Hep) formed the (G) second layer and (D and F) maintained the capability of regeneration. (H) However, ECs and other NPCs constituted the cover of the spheroid. (B and E) Around day 4-7, the sponge was observed. The ECs formed net structures to attach to the wall of the wells. ECs, endothelial cells; NPCs, non-parenchymal cells. 

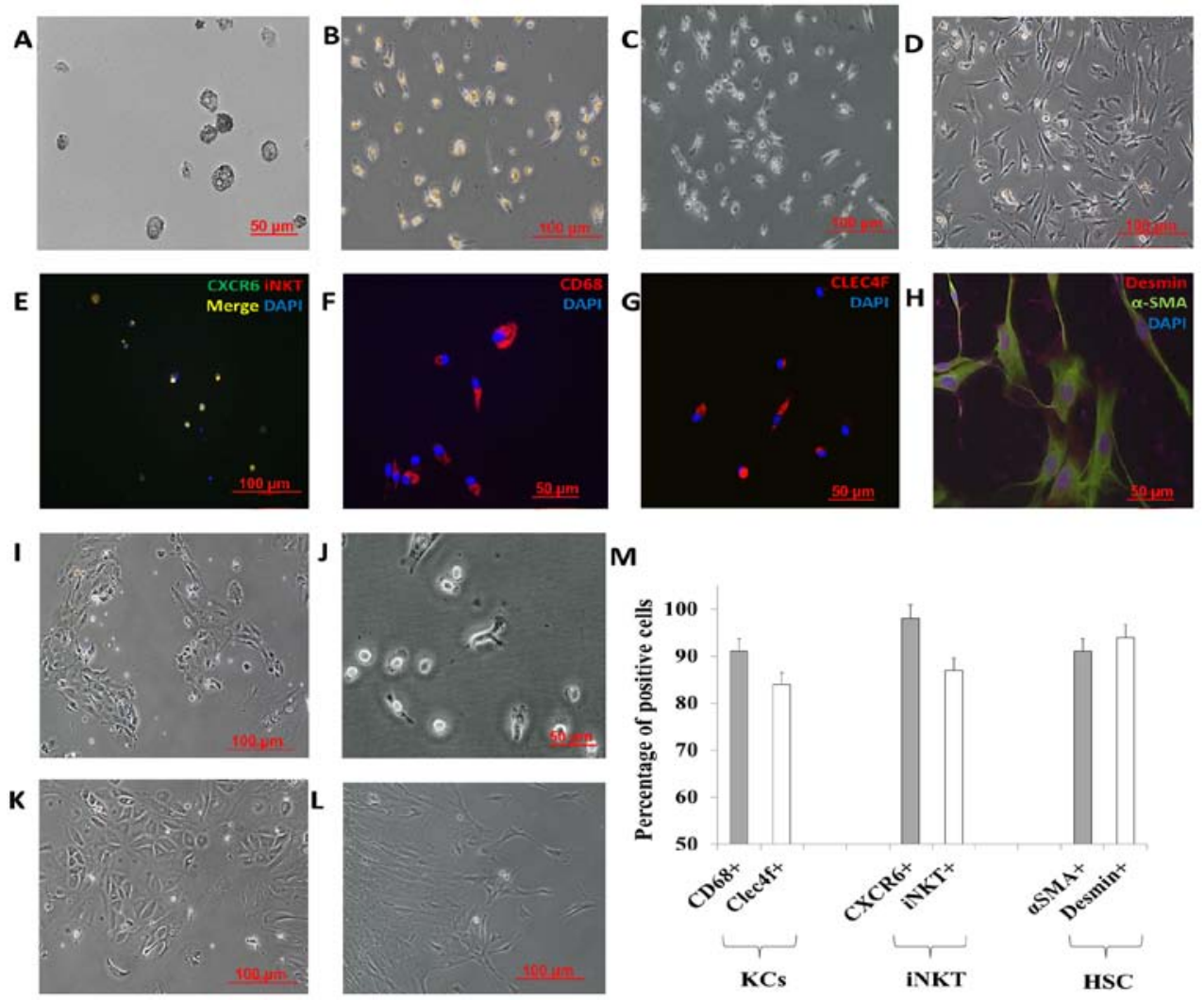

Figure 6. Morphology and staining of NPCs after isolation. (A) iNKT cells. (E) Immunophenotypic staining of iNKT cells with iNKT and CXCR6 antibodies. (B) Macrophages (black arrow, M1 macrophages; yellow arrow, M2 macrophages). (F) Immunophenotypic staining of macrophages. (C and G) KCs. (D and H) HSCs. (I) Intrahepatic biliary epithelial cells. (J) DCs. (K) Lymphatic vessel ECs. (L) Fibroblasts. (M) Purification (percentage) of KCs, iNKT cells and HSCs. NPCs, non-parenchymal cells; iNKT cells, invariant natural killer T cells; KCs, Kupffer cells; HSCs, hepatic stellate cells; DCs, dendritic cells; ECs, endothelial cells.
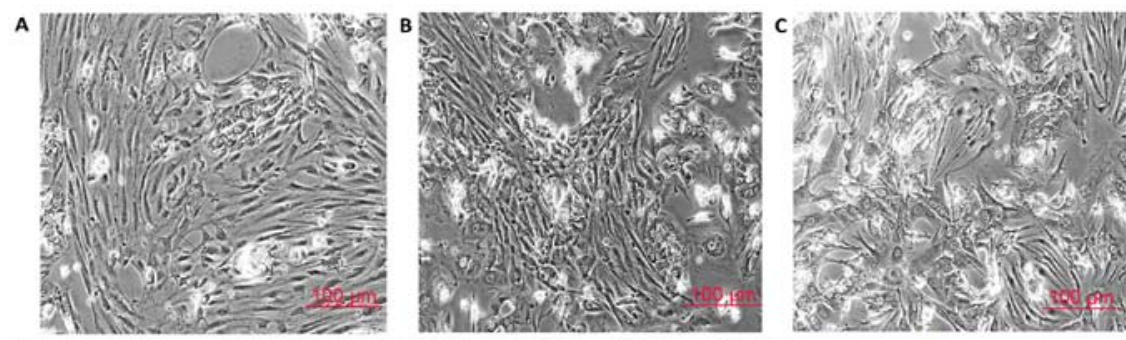

D
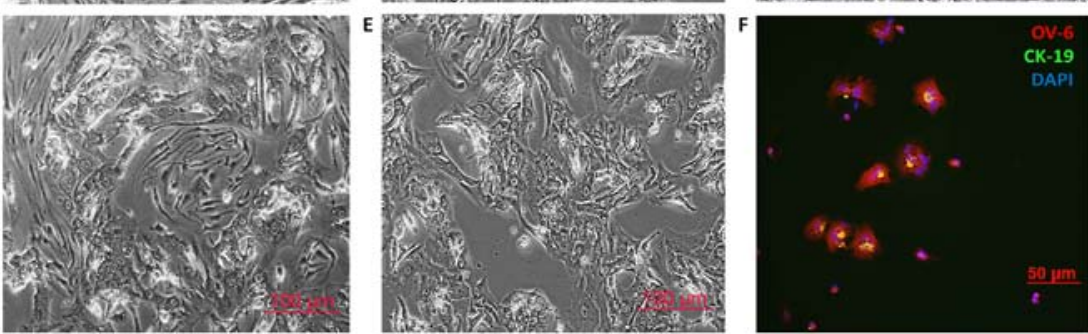

Figure 7. (F) Hepatic progenitor cell-like cells differentiated to hepatocytes surrounding the EC niches on (A) day 5, (B) day 6, (C) day 7, (D) day 8 and (E) day 10 were then separated from the LSECs. EC, endothelial cell; LSECs, liver sinusoid endothelial cells.

$100 \mathrm{U} / \mathrm{ml}$ penicillin, $0.1 \mathrm{mg} / \mathrm{ml}$ streptomycin, and $2 \mathrm{mM}$ L-glutamine. Freshly isolated HSCs exhibited the phenotypically star-shaped morphology and were transformed into an activated state, as identified by a myofibroblast-like phenotype within 10 days of cell culture. In the present study, HSCs were stained for $\alpha$-SMA, desmin and GFAP, well-known markers of HSCs to distinguish HSCs from myofibroblasts as described elsewhere (17). Imaging revealed high protein expression of desmin and GFAP in the HSCs. Most of the HSCs were $\alpha$-SMA-positive cells, which confirmed the identity of the activated HSCs. Intrahepatic human biliary epithelial cells (hBECs) were semi-purified by centrifugation on a Percoll gradient and were further immunopurified. hBECs were cytokeratin-7 $\left(\mathrm{CK}-7^{+}\right)$, CK-19 ${ }^{+}(18,19)$ and $\alpha$-SMA- cells. 
Hepatic progenitor cells were driven to differentiate into hepatoblasts. The oval cells were collected as described above. Cells were cultured using iPSC cell growth medium (Miltenyi Biotec $\mathrm{GmbH}$ ) with $10 \mathrm{ng} / \mathrm{ml}$ HGF. On day 0, the oval cells (hepatic progenitor cells) displayed small oval morphology with a dark nucleus and few cytoplasma. Immunostaining revealed that most of the cells were positive for the definitive markers CK-19 and OV-6 (Fig. 7F) and the hepatic progenitor cell marker EpCAM (data not shown) (20-22). On day 2 the oval cells started to display typical LSEC morphology, and LSECs were enriched on day 3-4. The number of LSECs reached maximal levels on day 4-6. Five to six days of iPSC cell growth medium and HGF-induction treatment resulted in the appearance of hepatocyte-like cells, which are reported to be hepatoblasts (Fig. 7A) (23). Fig. 7B-E shows the enrichment of the hepatoblasts on day 7-9.

\section{Discussion}

In order to improve the research platform for cancer research, especially for in vitro liver metastatic model establishment, functional and reproducible human liver cell co-culture models as well as high quality and quantity hepatocytes and non-parenchymal hepatic cells are essential. Recently, Pfeiffer et al isolated human hepatocytes and NPCs from tissue obtained by partial liver resection by a two-step EDTA/collagenase perfusion technique. The obtained cell fractions were purified by Percoll density gradient centrifugation. KCs, LSECs and HSCs were further separated using selective adherence to plastic. LSECs were separated by MACS using CD31 microBeads (24). Most recently, Werner et al established a three layer iodixanol density gradient using 9 and $12.6 \%$ iodixanol to separate NPCs. The advantage of the method of Werner et al is that LSECs and KCs can be separated as one fraction from the HSC population. These two methods were reported and approved to be available for high quality and quantity (25). In order to increase the purity, magnetic beads specific for CD31 or CD146 were used to label LSECs. Both methods provide cell yields comparable to those obtained by single and multiple cell type isolation methods.

In the present study, we established an efficient and co-culture isolation method based on $3 \mathrm{D}$ cell co-culture, long-term cell co-culture, density gradient centrifugation and MACS. The presented method provided high purities and comparable cell yields to the methods above.

Most recently, Lavin et al identified 12,743 macrophage-specific enhancers and established that tissue-resident macrophages have distinct enhancer landscapes beyond what can be explained by developmental origin. Clec4f was reported to be the biomarker of liver-resident macrophages, as known $\mathrm{KCs}$, which would be a specific marker for KC characteristics (13). In the present study, we found less $\mathrm{Clec}_{4} \mathrm{f}^{+} \mathrm{KCs}$ than $\mathrm{CD} 68^{+}$ macrophages. However, in the co-culture model, $87 \pm 6.3 \%$ of the isolated macrophages were Clec4f-positive, which might also be differentiated tissue-resident macrophages that were reprogrammed by the 3D cultured liver microenvironment.

The liver has a dual blood supply. The hepatic artery and portal vein both drain into the hepatic sinusoids, which represent the capillary network in the liver. The endothelial lining of liver sinusoids demonstrates microheterogeneity between periportal and centrilobular regions (26). Nolan et al identified LSECs as VEGFR3 ${ }^{+}$IsolectinGS $_{\mathrm{IB} 4}{ }^{+} \mathrm{CD} 34^{\mathrm{dim} /}{ }^{-} \mathrm{IgG}^{-}$cells (27). VE-cadherin ${ }^{+}$ESC $^{-}$ECs (embryonic stem cell differentiation into ECs) were found to consist of $\sim 60 \%$ of the vasculature in the regenerating liver in the same report. In line with this study, our study showed that both LSECs and microvascular ECs expressed VE-cadherin. To increase the purity of the LSECs, VEGFR $3{ }^{+}$IsolectinGS ${ }_{\mathrm{IB} 4}{ }^{+} \mathrm{CD} 34^{\mathrm{dim} /-}$ cell sorting would be recommended as an available strategy. Many groups used CD31 as a sorting strategy for LSECs $(28,29)$, however, it was reported that purified ESC-ECs maintained 99.3\% VE-cadherin and CD31 positivity for at least 4 weeks after purification (25).

With only $<1 \%$ of the NPC population as hepatic DCs, isolating a sufficient amount of hepatic DCs is a timely and costly task. Several methods using cytokine stimulation have been developed to increase the total hepatic DC population in vitro and in vivo (30). Joyee et al provide direct evidence that iNKTs preferentially promote the functional development of DCs (31). Bollyky et al also found that iNKT cell activation with $\alpha$-Galcer induced mature DCs (23). In this study, $\alpha$-Galcer, as a specific ligand for human and mouse natural killer T (NKT) cells, was added into the culture system of monocytes. We supposed that iNKT cells could be selectively activated so that the DCs, mostly monocyte-derived dendritic cells (moDCs) would be induced. However, we only separated $\mathrm{CD} \mathrm{c}^{+} \mathrm{DCs}$ in this study, which was the major subset of classic human DCs as well as inflammatory DCs (32). The subgroups of DCs need to be further analyzed by flow cytometry.

Concerning the biliary epithelial cells, their limited number (3\% of the total liver mass) and their intrahepatic localization have limited the development of in vitro cell models in order to gain molecular insights into their function (33). In the present study, we isolated hBECs from chronic liver injury, most of which were liver metastasis, as it was reported that chronic cell injury such as chronic hepatitis elicited mobilization of hepatic endodermal stem cells or oval cells, which can give rise both to hepatocytes and cholangiocytes (34).

Reports have suggested that a 3D liver cell co-culture system is a promising approach to mimic the tissue architecture in vivo $(35,36)$. Kostadinova et al (37) and Messner et al $(35)$ reported the earliest investigation of 3D co-culture liver model. Messner et al showed that the accumulation of hepatocytes and NPCs in hanging drops resulted in microtissue formation within 3 days. Immunohistochemical staining showed hepatocytes, $\mathrm{CD} 8^{+} \mathrm{KCs}$ as well as $\mathrm{CD} 31^{+} \mathrm{ECs}$. In addition, the functionality of liver microtissues was reported to be preserved for more than 5 weeks, as indicated by persistent albumin secretion. However, the PHHs in our hanging drop model did not attach after a 2-week cultivation (27). And the functionality of the PHHs was not as sufficient as reported. This might be because in our study, the cell number ( $>5,000$ cells) and diameter of the spheroids were larger than these parameters in the previous studies. It is recommended to investigate the functionality of NPCs and PHHs on organotypic co-culture models.

In conclusion, we developed an efficient and 3D cell co-culture isolation method. Isolated $\mathrm{KCs}$, sinusoid ECs, ECs, biliary epithelial cells, DCs, stellate cells, hepatic progenitor cell-like cells and iNKT cells can be further used as co-cultures for cancer research in particular tumor immunology and liver metastasis. 


\section{Acknowledgements}

We would like to thank Wolfgang E. Thasler, and Hepacult $\mathrm{GmbH}$ for their assistance with the hepatocyte isolation. This study was supported, in part, by the China Scholarship Council (CSC) (201306230127) and the German Academic Exchange Service scholarship.

\section{References}

1. Paget S: The distribution of secondary growths in cancer of the breast. 1889. Cancer Metastasis Rev 8: 98-101, 1989.

2. Langley RR and Fidler IJ: Tumor cell-organ microenvironment interactions in the pathogenesis of cancer metastasis. Endocr Rev 28: 297-321, 2007.

3. Wanebo HJ, Chu QD, Avradopoulos KA and Vezeridis MP: Current perspectives on repeat hepatic resection for colorectal carcinoma: A review. Surgery 119: 361-371, 1996.

4. Malafosse R, Penna C, Sa Cunha A and Nordlinger B: Surgical management of hepatic metastases from colorectal malignancies. Ann Oncol 12: 887-894, 2001.

5. Bentrem DJ, Dematteo RP and Blumgart LH: Surgical therapy for metastatic disease to the liver. Annu Rev Med 56: 139-156, 2005.

6. Pawlik TM, Schulick RD and Choti MA: Expanding criteria for resectability of colorectal liver metastases. Oncologist 13: 51-64, 2008.

7. Bhogal RH, Hodson J, Bartlett DC, Weston CJ, Curbishley SM, Haughton E, Williams KT, Reynolds GM, Newsome PN, Adams DH, et al: Isolation of primary human hepatocytes from normal and diseased liver tissue: A one hundred liver experience. PLoS One 6: e18222, 2011.

8. Seglen PO: Preparation of rat liver cells. 3. Enzymatic requirements for tissue dispersion. Exp Cell Res 82: 391-398, 1973.

9. Bhogal RH, Hodson J, Bartlett DC, Weston CJ, Curbishley SM, Haughton E, Williams KT, Reynolds GM, Newsome PN, Adams DH, Afford SC. Isolation of primary human hepatocytes from normal and diseased liver tissue: A one hundred liver experience. PloS one 6: e18222, 2011.

10. Rothe GM (ed). Electrophoresis of Enzymes: Laboratory Methods (Springer Lab Manuals). 1st edition. Springer-Verlag, Berlin, Heidelberger, pp39-45, 1994.

11. Holmén C, Christensson M, Pettersson E, Bratt J, Stjärne P, Karrar A and Sumitran-Holgersson S: Wegener's granulomatosis is associated with organ-specific antiendothelial cell antibodies. Kidney Int 66: 1049-1060, 2004.

12. Isidro RA, Bonilla FJ, Pagan H, Cruz ML, Lopez P, Godoy L, Hernandez S, Loucil-Alicea RY, Rivera-Amill V, Yamamura Y, et al: The probiotic mixture VSL\#3 alters the morphology and secretion profile of both polarized and unpolarized human macrophages in a polarization-dependent manner. J Clin Cell Immunol 5: 1000227, 2014.

13. Lavin Y, Winter D, Blecher-Gonen R, David E, Keren-Shaul H, Merad M, Jung S and Amit I: Tissue-resident macrophage enhancer landscapes are shaped by the local microenvironment. Cell 159: 1312-1326, 2014.

14. Daneker GW, Lund SA, Caughman SW, Swerlick RA, Fischer AH, Staley CA and Ades EW: Culture and characterization of sinusoidal endothelial cells isolated from human liver. In Vitro Cell Dev Biol Anim 34: 370-377, 1998.

15. Elvevold K, Smedsrød B and Martinez I: The liver sinusoidal endothelial cell: A cell type of controversial and confusing identity. Am J Physiol Gastrointest Liver Physiol 294: G391-G400, 2008.

16. Podgrabinska S, Braun P, Velasco P, Kloos B, Pepper MS and Skobe M: Molecular characterization of lymphatic endothelial cells. Proc Natl Acad Sci USA 99: 16069-16074, 2002.

17. Yin C, Evason KJ, Asahina K, Stainier DY. Hepatic stellate cells in liver development, regeneration, and cancer. J Clin Invest. 123: 1902-1910, 2013

18. Massani M, Stecca T, Fabris L, Caratozzolo E, Ruffolo C, Furlanetto A, Morton S, Cadamuro M, Strazzabosco M and Bassi N: Isolation and characterization of biliary epithelial and stromal cells from resected human cholangiocarcinoma: A novel in vitro model to study tumor-stroma interactions. Oncol Rep 30: 1143-1148, 2013.
19. Muff MA, Masyuk TV, Stroope AJ, Huang BQ, Splinter PL, Lee SO and Larusso NF: Development and characterization of a cholangiocyte cell line from the PCK rat, an animal model of Autosomal Recessive Polycystic Kidney Disease. Lab Invest 86: 940-950, 2006.

20. Duret C, Gerbal-Chaloin S, Ramos J, Fabre JM, Jacquet E, Navarro F, Blanc P, Sa-Cunha A, Maurel P and Daujat-Chavanieu M: Isolation, characterization, and differentiation to hepatocyte-like cells of nonparenchymal epithelial cells from adult human liver. Stem Cells 25: 1779-1790, 2007.

21. Terrace JD, Currie IS, Hay DC, Masson NM, Anderson RA, Forbes SJ, Parks RW and Ross JA: Progenitor cell characterization and location in the developing human liver. Stem Cells Dev 16: 771-778, 2007.

22. Li J, Xin J, Zhang L, Wu J, Jiang L, Zhou Q, Li J, Guo J, Cao H and Li L: Human hepatic progenitor cells express hematopoietic cell markers CD45 and CD109. Int J Med Sci 11: 65-79, 2013.

23. Bollyky PL and Wilson SB: CD1d-restricted T-cell subsets and dendritic cell function in autoimmunity. Immunol Cell Biol 82: 307-314, 2004.

24. Pfeiffer E, Kegel V, Zeilinger K, Hengstler JG, Nüssler AK, Seehofer D and Damm G: Isolation, characterization, and cultivation of human hepatocytes and non-parenchymal liver cells. Exp Biol Med (Maywood) 240: 645-656, 2015.

25. Werner M, Driftmann S, Kleinehr K, Kaiser GM, Mathé Z, Treckmann JW, Paul A, Skibbe K, Timm J, Canbay A, et al All-in-one: Advanced preparation of human parenchymal and non-parenchymal liver cells. PloS One 10: e0138655, 2015.

26. Aird WC: Phenotypic heterogeneity of the endothelium: II. Representative vascular beds. Circ Res 100: 174-190, 2007.

27. Nolan DJ, Ginsberg M, Israely E, Palikuqi B, Poulos MG, James D, Ding BS, Schachterle W, Liu Y, Rosenwaks Z, et al: Molecular signatures of tissue-specific microvascular endothelial cell heterogeneity in organ maintenance and regeneration. Dev Cell 26: 204-219, 2013.

28. Asahara T, Murohara T, Sullivan A, Silver M, van der Zee R, Li T, Witzenbichler B, Schatteman G and Isner JM: Isolation of putative progenitor endothelial cells for angiogenesis. Science 275: 964-967, 1997

29. Duncan GS, Andrew DP, Takimoto H, Kaufman SA, Yoshida H, Spellberg J, de la Pompa JL, Elia A, Wakeham A, Karan-Tamir B, et al: Genetic evidence for functional redundancy of platelet/endothelial cell adhesion molecule-1 (PECAM-1): CD31-deficient mice reveal PECAM-1-dependent and PECAM-1-independent functions. J Immunol 162: 3022-3030, 1999.

30. Hsu W, Shu SA, Gershwin E and Lian ZX: The current immune function of hepatic dendritic cells. Cell Mol Immunol 4: 321-328, 2007.

31. Joyee AG, Uzonna $J$ and Yang $X$ : Invariant NKT cells preferentially modulate the function of $\mathrm{CD} 8 \alpha^{+}$dendritic cell subset in inducing type 1 immunity against infection. J Immunol 184: 2095-2106, 2010.

32. Collin M, McGovern N and Haniffa M: Human dendritic cell subsets. Immunology 140: 22-30, 2013.

33. Dianat N, Dubois-Pot-Schneider H, Steichen C, Desterke C, Leclerc P, Raveux A, Combettes L, Weber A, Corlu A and Dubart-Kupperschmitt A: Generation of functional cholangiocyte-like cells from human pluripotent stem cells and HepaRG cells. Hepatology 60: 700-714, 2014.

34. Forbes S, Vig P, Poulsom R, Thomas H and Alison M: Hepatic stem cells. J Pathol 197: 510-518, 2002.

35. Messner S, Agarkova I, Moritz W and Kelm JM: Multi-cell type human liver microtissues for hepatotoxicity testing. Arch Toxicol 87: 209-213, 2013.

36. Schyschka L, Sánchez JJ, Wang Z, Burkhardt B, Müller-Vieira U, Zeilinger K, Bachmann A, Nadalin S, Damm G and Nussler AK: Hepatic 3D cultures but not 2D cultures preserve specific transporter activity for acetaminophen-induced hepatotoxicity. Arch Toxicol 87: 1581-1593, 2013.

37. Kostadinova R, Boess F, Applegate D, Suter L, Weiser T, Singer T, Naughton B and Roth A: A long-term three dimensional liver co-culture system for improved prediction of clinically relevant drug-induced hepatotoxicity. Toxicol Appl Pharmacol 268: 1-16, 2013 . 